\title{
Role of promoter element in $c-m p l$ gene expression induced by TPO
}

\author{
By
}

\author{
Masataka SUNOHARA ${ }^{1,2}$, Shigeru MORIKAWA ${ }^{3}$, Akira FUSE ${ }^{2}$, and Iwao SATO ${ }^{1}$ \\ ${ }^{1}$ Department of Anatomy, School of Life Dentistry at Tokyo, The Nippon Dental University, \\ 1-9-20 Fujimi, Chiyoda-ku, Tokyo 102-8159, Japan \\ ${ }^{2}$ Department of Safety Research on Blood Products and Biologics, National Institute of Infectious Diseases, \\ 4-7-1 Gakuen, Musashimurayama, Tokyo 208-0011, Japan \\ ${ }^{3}$ Department of Veterinary, Science National Institute of Infectious Diseases, \\ 1-23-1 Toyama, Shinjuku-ku, Tokyo 162-8640, Japan
}

- Received for Publication, December 28, $2012-$

Key Words: thrombopoietin (TPO), c-mpl, protein kinase C (PKC), megakaryocyte, CMK

\begin{abstract}
Summary: Thrombopoietin (TPO) and its receptor, c-Mpl, play the crucial role for the development of megakaryocyte and considered to regulate megakaryocytopoiesis. Previously we reported that TPO increased the $c$-mpl promoter activity determined by a transient expression system using a vector containing the luciferase gene as a reporter and the expression of the $c$-mpl gene is modulated by transcription through a protein kinase $\mathrm{C}$ (PKC)-dependent pathway in the megakaryoblastic cells. In this research, to elucidate the required elements in $c$-mpl promoter, the promoter activity of the deletion constructs and site-directed mutagenesis were measured by a transient transfection assay system. Destruction of -77GATA in $c$-mpl promoter decreased the activity by $\mathbf{2 2 . 8 \%}$. Our study elucidated that -77GATA involved in TPO-induced $c$-mpl gene expression in a human megakaryoblastic cell line, CMK.
\end{abstract}

\section{Introduction}

Thrombopoietin (TPO) and its receptor, c-Mpl, are required for megakaryopoiesis and platelet development (Lok et al.,19941), De Sauvage et al.,19942), Wendling et al.,19943), Kaushansky ,19994) $)$. PKC is a member of a family of serine/threonine protein kinases in the cytosol involved in pleiotropic processes such as cytokine secretion, cell growth and differentiation (Ohno et al.,19915), Nishizuka et al.,19926) $)$. CMK, the human megakaryoblastic cell line, was established from the peripheral cells obtained from a patient with acute megakaryoblastic leukemia and Down' syndrome (Komatsu et al.,19897), Sato et al.,19898) $)$. Previously we characterized an expression of c-Mpl on the CMK cells (Sato et al.,19898) and found that TPO down-regulated the expression of c-Mpl on the cell surface (Sato et al.,19989)). TPO has also been shown to activate PKC in the CMK cells (Hong et al., 199810)). Recently we suggested that TPO-induced $c-m p l$ promoter activity was modulated at transcription level through a PKC-dependent pathway in the CMK cells (Sunohara et al.,200311), 200412)). In this research, we investigated the required promoter elements in $c-m p l$ gene expression induced by TPO.

\section{Materials and methods}

\section{Cell Culture}

The CMK cell line was established from a patient with acute megakaryoblastic leukemia and Down' syndrome (Komatsu et al.,19897), Sato et al.,19898)). As described previously (Sunohara et al., 200311), 200913), 201014), 200815)), the cells were cultured in RPMI 1640 medium (GIBCO, Grand Island, NY) containing 10\% FCS, 2mM L-glutamine, 100 units $/ \mathrm{ml}$ penicillin, $100 \mu \mathrm{g} / \mathrm{ml}$ streptomycin, and $0.25 \mu \mathrm{g} / \mathrm{ml}$ amphotericin $\mathrm{B}$ at $37^{\circ} \mathrm{C}$ in humid air containing $5 \% \mathrm{CO}_{2}$ atmosphere. $\mathrm{CMK}$ cells were cultured in RPMI 1640 medium (GIBCO, Grand Island, NY) supplemented with $10 \%$ fetal calf serum (FCS), $2 \mathrm{mM}$ L-glutamine, 100 units $/ \mathrm{ml}$ penicillin, $100 \mu \mathrm{g} / \mathrm{ml}$ streptomycin, and $0.25 \mu \mathrm{g} / \mathrm{ml}$ amphotericin $\mathrm{B}$ at $37^{\circ} \mathrm{C}$ in humidified air containing $5 \% \mathrm{CO}_{2}$ atmosphere. 


\section{Reagents}

Protein kinase C modulator, GF 109203 (CALBIOCHEM Co., La Jolla, CA) was dissolved in DMSO as a stock solution and kept at $-80^{\circ} \mathrm{C}$ until use. Control medium included DMSO at the highest concentration used in stock solutions did not change $c-m p l$ gene expression.

Thrombopoietin (Recombinant human TPO) was purified as previously described (De Sauvage et al.,19942)).

Construction of a reporter plasmid to measure the c-mpl-promoter activity.

The construct[pGL3-c-mpl (-310)] has been reported previously (Sunohara et al., 200311), 200913) 201014), the $310 \mathrm{bp}$ upstream promoter region of $c-m p l$ was amplified from the chromosomal DNA of the CMK cells by polymerase chain reaction using the following primer set, (forward 5'-CTA TTC TGA TGC TAT TAT ACC ATT C-3', reverse 5'-CTT GGC TGC TGA CTT GGG C-3'). The PCR product was cloned into pGEM-Teasy vector (Promega, Madison, WI) and designated as pGEM-c-mplprom. For the determination of the promoter activity of $c$-mpl, a $B g l$ II /Hind III fragment of pGEM-c-mpl-prom was subcloned into a defined $B g l$ II /Hind III site of the pGL3-basic vector (Promega) to construct pGL3-c-mpl $(-310)$ in which the luciferase gene was driven under control of the $c-m p l$ promoter (Fig.1).

\section{Promoter deletion constructs}

As described previously (Sunohara et al., 200815), the truncated forms of the $310 \mathrm{bp}$ upstream promoter region of $c$ - $m p l$ were amplified from the pGEM-c-mpl-prom by polymerase chain reactions using the following primer sets: $c$-mpl-prom (-121) (forward: 50-GGA TGG GCC CCA GA-30, reverse: 50-CTT GGC TGC TGA CTT GGG C-30), $c$-mpl prom (-83) (forward: 50-GGG GCT GTATCT GAC-30, reverse:50-CTT GGC TGC TGA CTT GGG C-30). The PCR products were cloned into pGEM-Teasy vector and their sequences were confirmed. Then their Bgl II/Hind III fragments were subcloned into the Bgl II/Hind III site of the pGL3-basic vector to construct pGL3-c-mpl (-121), pGL3-c-mpl (-83), respectively (Fig. 2A). In these constructs, the luciferase gene is driven under control of the truncated forms of $c-m p l$ promoter.

\section{Site-directed mutagenesis}

As reported previously (Sunohara et al., 200815)), to examine the positive regulatory elements in the $c-m p l$ promoter, we examined the effect of a mutation within the positive regulatory motifs on the promoter activity (Fig. 3A). The promoter activity of the mutated construct (pGL3-77GATA (-)) was measured after transient transfection assay in CMK cells. The mutation destroyed the GATA sequence (GATA $\rightarrow$ GCTA) in pGL3-77GATA (-) (mutated residue is underlined).

\section{Transient transfection assay}

As described previously(Sunohara et al., 200311), 200913), 201014)), three reporter plasmids, pGL3-basic vector (as a negative control), pGL3-control vector in which the luciferase gene is driven under SV40 promoter (as a positive control, Promega) and the pGL3-c-mpl (-310) were used for transfection assay. A plasmid, pRL-CMV (TOYO INC MFG CO., LTD., Tokyo), which expresses Renilla (SeaPansy) luciferase under control of CMV early promoter, was used to normalize the transfection efficiency. PMA (20nM) was added to the CMK cells and then the cells were washed twice in PBS. The reporter plasmids $(1 \mu \mathrm{g})$ were co-transfected with the pRL-CMV into the CMK cells for $5 \mathrm{~h}$ by a lipofection method using the DMRIE-C reagent (GIBCO, Grand Island, NY) according to the manufacturer's instructions. Then, the cells were cultured in the growth medium (RPMI1640, $10 \% \mathrm{FCS}$ ) for $19 \mathrm{~h}$, washed twice in PBS, lyzed in a cell lysis reagent, LCß (TOYO INC MFG CO., LTD., Tokyo) and stored at $-80{ }^{\circ} \mathrm{C}$ until luciferase assay. The luciferase activity was quantified by using a PicaGene-Dual-SeaPansy Luminescence Kit (TOYO INC MFG CO., LTD., Tokyo). Twenty $\mu$ l aliquot of cell lysates were added to $100 \mu \mathrm{l}$ of luciferase reaction mixture supplied in the kit, and luciferase activity was measured with a luminometer in accordance with the manufacturer's instructions. The cell lysate of the cells transfected with a promoter-less control vector, pGL3-Basic Vector, was used as a negative control and the SeaPansy luciferase activity expressed from pRL-CMV was used to evaluate transfection efficiency and to normalize luciferase activity in all lysates. Results are expressed as the percentage of the control values. All the experiments were performed at least three times, and results represent means \pm SD of at least 3 measurements.

\section{Results}

We examined the effect of TPO on $c-m p l$ promoter activity by using transient transfection assay system. Besides pGL3-c-mpl (-310), we also examined the effect of the deletion constructs and site-directed mutagenesis on TPO-induced $c-m p l$ promoter activity at the same time.

Effect of TPO and protein kinase C inhibitor on the activation of the c-mpl promoter

After pretreatment with TPO (100 ng/ml for $3 \mathrm{~h}$.), TPO increased the $c$ - $m p l$ promoter activity and PKC inhibitor (GF109303X) reduced the basal level of the $c-m p l$ promoter activity. As reported previously (Sunohara et al.,200311), 200815)), we confirmed again that PKC plays the key role in maintaining $c-m p l$ transcription in $\mathrm{CMK}$ cells (data not shown). 
Fig. 1. Schematic representation of pGL3-c-mpl (-310) pGL3-c-mpl (-310) is represented in Fig. 1.

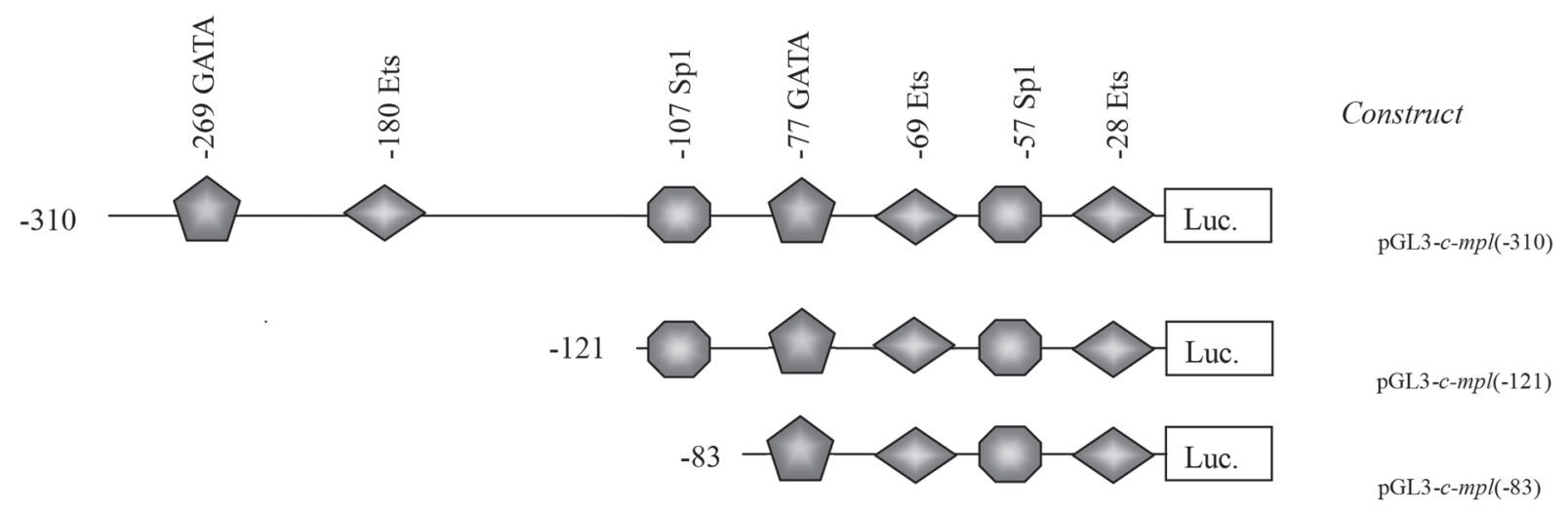

Fig. 2A. Schematic representation of putative regulatory elements in the $c-m p l$ promoter and the organization of these sites in the deletion constructs. To determine the suitable construct for hight-level specific expression, we examined the three deletion construts. Each deletion construct is represented in Fig. 2A.

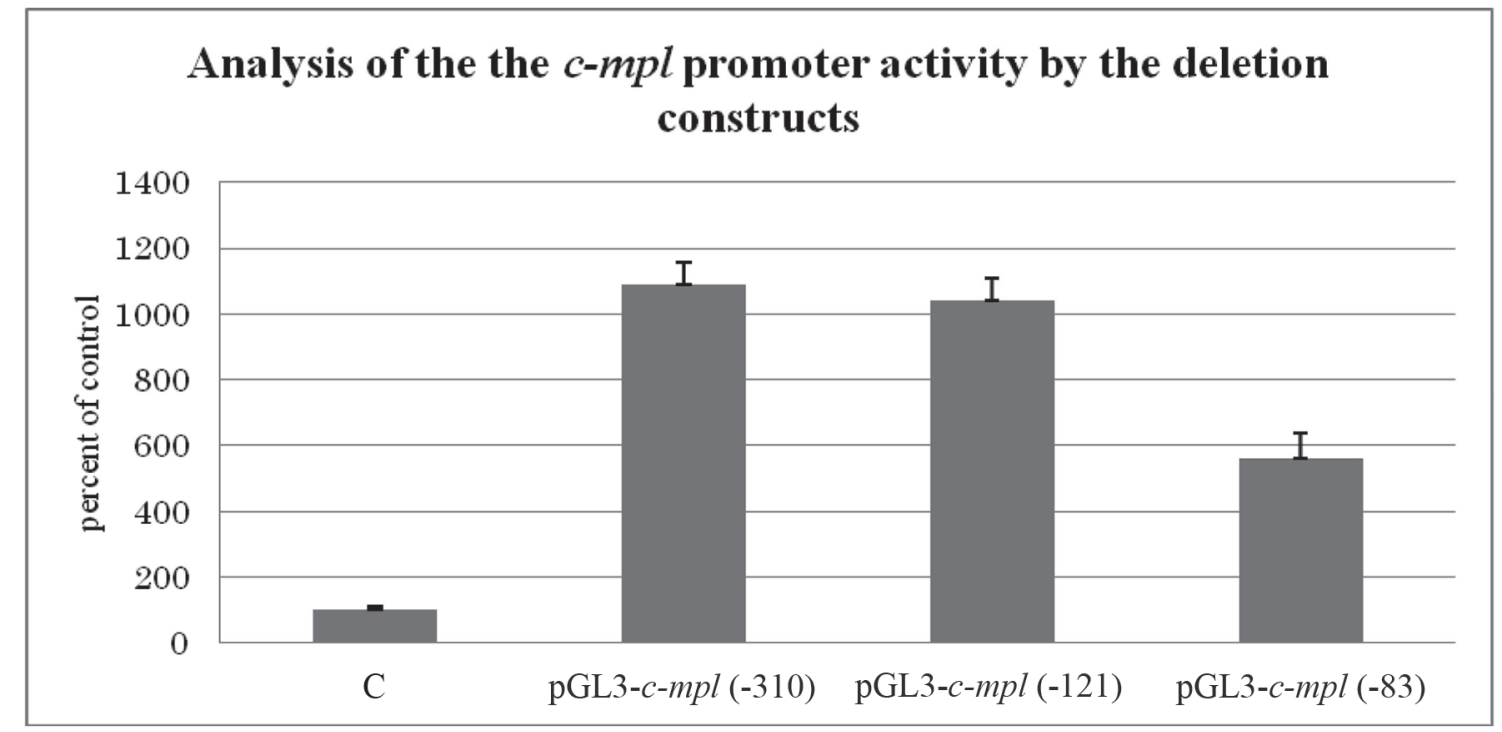

Fig. 2B. Analysis of the $c-m p l$ promoter activity by the deletion constructs. The activity of the each deletion construct (pGL3-c-mpl(-310), pGL3c-mpl(-121), pGL3-c-mpl(-83) was measured by transient transfection assay in CMK cells (Fig. 2B). Results are expressed as the percent of the control values. All experiments were performed at least three times and results represent means $\pm \mathrm{SD}$ of at least 3 measurements. 


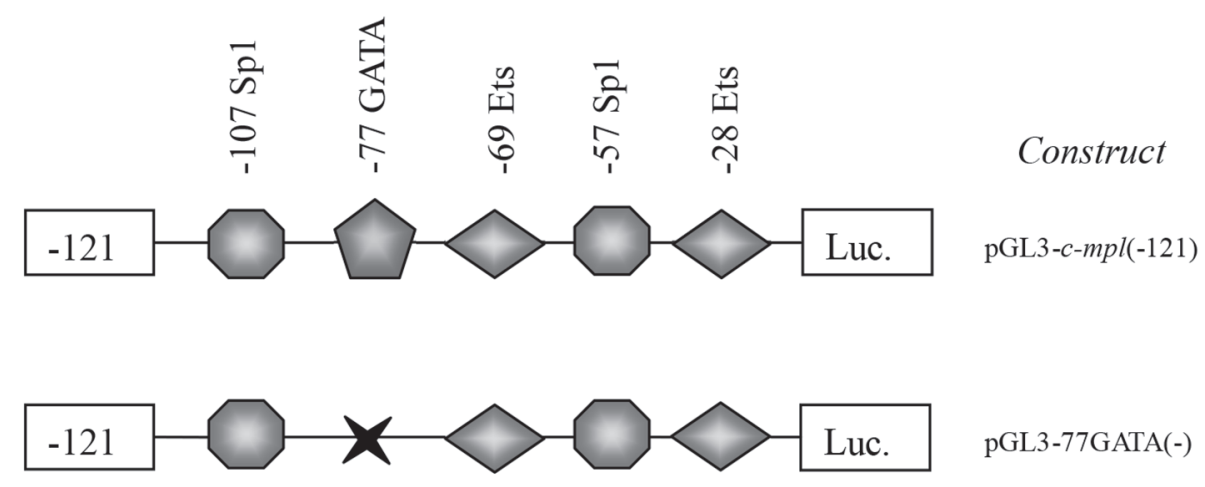

Fig. 3A. Schematic representation of the organization of putative regulatory elements in the mutated construct. Schematic representation of putative regulatory elements in pGL3-c-mpl(121) and the organization of these sites in the mutated constructs. To examine the positive regulatory elments in the $c-m p l$ promoter, we examined the effect of a mutation within the positive regulatory motifs on the promoter activity. The mutation within the positive regulatory motif(-77GATA) is represented in.Fig. 3A. The crosses $(\mathcal{W})$ designate the mutated region.

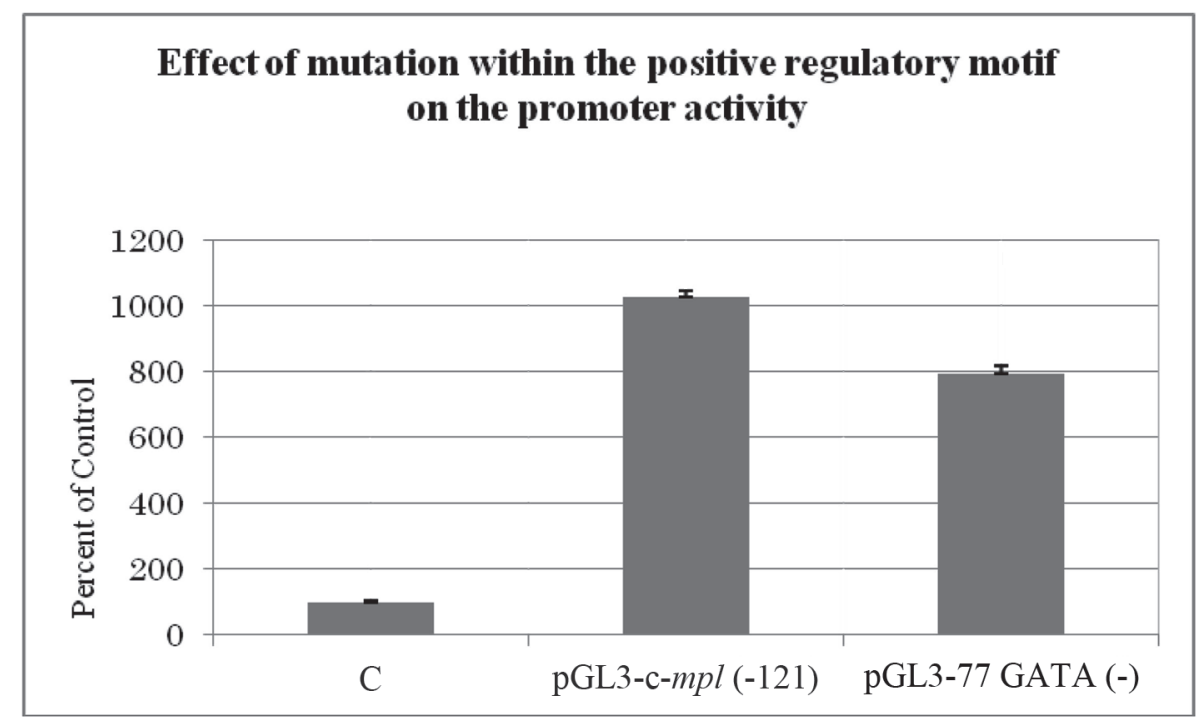

Fig. 3B. Effect of mutation within the positive regulatory motif on the promoter activity The promoter activity of the mutated construct [pGL377GATA(-)] was measured by transient transfection assay in CMK cells (Fig. 3B). Results are expressed as the percent of the control values. All the experiments were performed at least three times and results represent means $\pm \mathrm{SD}$ of at least 3 measurements.

Effect of deletions in regulatory elements on c-mpl promoter activity

As to the deletion constructs, the activity was clearly reduced from pGL3-c-mpl (-83) and we confirmed that pGL3-c-mpl $(-121)$ is the most suitable construct for the following our experiments (Fig. 2B).

Effect of point mutations in pGL3-c-mpl (-121) on the c-mpl promoter activity

To examine the positive regulatory elements in the $c-m p l$ promoter, we examined the effect of the mutation within the positive regulatory motifs on the promoter activity. The promoter activity of the mutated construct (pGL3-77GATA) was measured after transient transfection assay in CMK cells. As shown in Fig. 3B, destruction of $-77 \mathrm{GATA}$ in $c-m p l$ promoter decreased the activity by $22.8 \%$.

\section{Discussion}

We have already reported that pretreatment with TPO inhibits the binding activity of TPO to its receptor and led to downregulation of the number of $\mathrm{c}-\mathrm{Mpl}$ on the 
cell surface. PMA has also induced downregulation of $\mathrm{c}-\mathrm{Mpl}$ on CMK cells. These downregulations of c-Mpl were transient (Sato et al., 19989)). Recently we clarified that the promoter activity of $c-m p l$ was upregulated by TPO pretreatment and TPO-induced $c-m p l$ promoter activity was modulated at transcription level through a PKC-dependent pathway in the CMK cells (Sunohara et al.,200311), 200412)). In this research, we investigated the required promoter element in $c-m p l$ gene expression induced by TPO. The data of the promoter activity of the deletion constructs upon TPO treatment clearly showed that the important positive regulatory elements appeared to be at position less than -121 in $c-m p l$ promoter. So we confirmed that pGL3-c-mpl (-121) is the most suitable construct for the following our experiments (Fig. 2B).

To examine the positive regulatory elements in the $c-m p l$ promoter, the promoter activity of the mutated construct (pGL3-77GATA) was measured after transient transfection assay in CMK cells and destruction of -77GATA in c-mpl promoter decreased the activity by $22.8 \%$ (Fig. 3B).

It has been reported that the glycoprotein IIb promoter contains a specific erythromegakaryocytic enhancer that may interact with GATA-1 in megakaryocytic cells (Prandini et al., 199216)). And also GATA and Ets recognition sequences are an important determinant of megakaryocytic gene expression (Lemarchandel et al., 199317)). On the other hands, GATA-1-induced upregulation of $c-m p l$ is involved in erythroid and megakaryocytic differentiation in the murine myeloid cell line (Yamaguchi et al., 199818)) and GATA-1 serves multiple functions during megakaryocyte development (Vyas et al.,199919)).

Our study clearly defines that-77GATA was important as a positive regulatory element and involved in $c-m p l$ gene expression induced by TPO.

Further studies are needed to elucidate the required motifs for $c-m p l$ gene expression induced by TPO in human megakaryocytic cells.

\section{Acknowledgments}

This work was supported by a grant from the Ministry of Education, Science Sports and Culture of Japan (No. 10771001) and by a Grant-in-Aid for the Encouragement of Young Scientists (No. 13771085) from the Japan Society for the Promotion of Science (JSPS).

\section{References}

1) Lok S, Kaushansky K, Holly RD, Kuijper JL, Lofton-Day CE, Oort PJ, Grant FJ, Heipel MD, Burkhead SK, Kramer JM, et al: Cloning and expression of murine thrombopoietin cDNA and stimulation of platelet production in vivo. Nature 1994; 369:519-20.

2) De Sauvage FJ, Hass PE, Spencer SD, Malloy BE, Gurney AL, Spencer SA, Darbonne WC, Henzel WJ, Wong SC, Kuang WJ, et al: Stimulation of megakaryocytopoiesis and thrombopoiesis by the c-Mpl ligand. Nature 1994; 369:533-8.

3) Wendling F, Maraskovsky E, Debili N, Florindo C, Teepe M, Titeux $\mathrm{M}$, Methia N, Breton-Gorius J, Cosman D, Vainchenker W: c-Mpl ligand is a humoral regulator of megakaryocytopoiesis. Nature 1994; 369:571-4.

4) Kaushansky K: The enigmatic megakaryocyte gradually reveals its secrets. Bioessays 1999; 21:353-60.

5) Ohno S, Akita Y, Hata A, Osada S, Kubo K, Konno Y, Akimoto $\mathrm{K}$, Mizuno K, Saido T, Kuroki T, et al: Structural and functional diversities of a family of signal transducing protein kinases, protein kinase $\mathrm{C}$ family: two distinct classes of PKC, conventional cPKC and novel nPKC. Adv Enzyme Regul 1991; 31:287-303.

6) Nishizuka Y: Intracellular signaling by hydrolysis of phospholipids and activation of protein kinase C. Science 1992; 258:607-14.

7) Komatsu N, Suda T, Moroi M, Tokuyama N, Sakata Y, Okada M, Nishida T, Hirai Y, Sato T, Fuse A, et al: Growth and differentiation of a human megakaryoblastic cell line CMK. Blood 1989; 74:42-8.

8) Sato T, Fuse A, Eguchi M, Hayashi Y, Ryo R, Adachi M, Kishimoto Y, Teramura M, Mizoguchi H, Shima Y, et al: Establishment of a human leukaemic cell line (CMK) with megakaryocytic characteristics from a Down's syndrome patient with acute megakaryoblastic leukemia. Br J Haematol 1989; 72:184-90.

9) Sato T, Fuse A, Niimi H, Fielder PJ, Avraham H: Binding and regulation of thrombopoietin to human megakaryocytes. Br J Haematol 1998; 100:704-11.

10) Hong Y, Dumenil D, van der Loo B, Goncalves F, Vainchenker W, Erusalimsky JD: Protein kinase $\mathrm{C}$ mediates the mitogenic action of thrombopoietin in c-Mpl-expressing UT-7 cells. Blood 1998; 91(3):813-22.

11) Sunohara M, Morikawa S, Sato T, Sato I, Sato T, Fuse A: Modulation of human $c-m p l$ gene expression by thrombopoietin through protein kinase C. Cell Mol Biol 2003; 49. Online Pub: OL393-8.

12) Sunohara M, Sato I, Sato T, Fuse A: Modulation mechanism of c-mpl gene expression in human megakaryoblastic CMK cells. Dentistry in Japan 2004; 40. 3-6.

13) Sunohara M, Morikawa S, Murata H, Fuse A, Sato I:Modulation mechanism of c-Mpl promoter activity in megakaryoblastic cells. Okajimas Folia Anat Jpn. 2009 Nov; 86(3):89-91.

14) Sunohara M, Morikawa S, Murata H, Fuse A, Sato I: PKC plays a crucial roles in c-mpl gene expression in megakaryoblastic cells. Okajimas Folia Anat Jpn. 2010 Nov; 87(3):151-4.

15) Sunohara M, Morikawa S, Sato T, Miyado M, Sato I, Sato T, Fuse A. Promoter regulatory motifs involved in $c-m p l$ gene expression induced by PMA. Cell Biol Int. 2008; 32(6):692-7.

16) Prandini MH, Uzan G, Martin F, Thevenon D, Marguerie G. Characterization of a specific erythromegakaryocytic enhancer within the glycoprotein IIb promoter. J Biol Chem 1992; 267:10370e4.

17) Lemarchandel V, Ghysdael J, Mignotte V, Rahuel C, Romeo PH. GATA and Ets cis-acting sequences mediate megakaryocyte-specific expression. Mol Cell Biol 1993; 13:668e76.

18) Yamaguchi Y, Zon LI, Ackerman SJ, Yamamoto M, Suda T. Forced GATA-1 expression in the murine myeloid cell line M1: induction of c-Mpl expression and megakaryocytic/erythroid differentiation. Blood 1998; 91:450e7.

19) Vyas P, Ault K, Jackson CW, Orkin SH, Shivdasani RA. Consequences of GATA-1 deficiency in megakaryocytes and platelets. Blood 1999; 93:2867e75. 